\title{
DNA Mismatch Repair System: Repercussions in Cellular Homeostasis and Relationship with Aging
}

\author{
Juan Cristóbal Conde-Pérezprina, Miguel Ángel León-Galván, and Mina Konigsberg
}

Universidad Autónoma Metropolitana Unidad Iztapalapa, 186 Avenida San Rafael Atlixco, 09340 Mexico City, DF, Mexico

Correspondence should be addressed to Mina Konigsberg, mkf@xanum.uam.mx

Received 31 May 2012; Revised 24 September 2012; Accepted 8 October 2012

Academic Editor: William C. Burhans

Copyright ( 2012 Juan Cristóbal Conde-Pérezprina et al. This is an open access article distributed under the Creative Commons Attribution License, which permits unrestricted use, distribution, and reproduction in any medium, provided the original work is properly cited.

\begin{abstract}
The mechanisms that concern DNA repair have been studied in the last years due to their consequences in cellular homeostasis. The diverse and damaging stimuli that affect DNA integrity, such as changes in the genetic sequence and modifications in gene expression, can disrupt the steady state of the cell and have serious repercussions to pathways that regulate apoptosis, senescence, and cancer. These altered pathways not only modify cellular and organism longevity, but quality of life ("health-span"). The DNA mismatch repair system (MMR) is highly conserved between species; its role is paramount in the preservation of DNA integrity, placing it as a necessary focal point in the study of pathways that prolong lifespan, aging, and disease. Here, we review different insights concerning the malfunction or absence of the DNA-MMR and its impact on cellular homeostasis. In particular, we will focus on DNA-MMR mechanisms regulated by known repair proteins MSH2, MSH6, PMS2, and MHL1, among others.
\end{abstract}

\section{Introduction}

Throughout their lives, organisms are exposed to many different environmental and internal stimuli that affect or modify their functionality. Aging has been considered an important phenomenon that is promoted or altered by these factors. The aging theory, proposed by Harman [1], establishes that unrepaired oxidative damage to biomolecules caused by free radicals and accumulated during an organism's lifetime, might bring on the aging process. Based on in vitro and in vivo analysis, it has been established that cellular metabolic pathways, like mitochondrial respiration, generates oxidants that can induce biomolecules oxidation [2]. Prolonged exposure to pollutants, metals, toxic compounds, and ionizing radiation, can also induce degenerative processes associated with premature aging $[3,4]$. When oxidative damage occurs, in particular DNA damage, it creates a cascade of events that may contribute to aging and disease [5]. However, cells have different mechanisms to maintain DNA biochemical integrity and stability. One of them is the base excision repair (BER) system, a cellular mechanism that repairs damaged bases in the DNA sequence originating from deamination, oxidation, and alkylation. Nucleotide excision repair (NER) is another DNA repair mechanism which differs from BER. While BER repairs individual damaged bases, the NER system corrects larger portions of DNA damage by removing the strand section that contains a major nucleotide lesion [6-8]. Both BER and NER systems are constantly active in the cell, repairing DNA damage as it is detected. However, there are other mechanisms that are activated during specific cellular stages, such as DNA mismatch repair system (MMR).

\section{DNA Mismatch Repair Proteins}

DNA MMR is an evolutionarily conserved process that corrects base mismatches generated during DNA replication that have escaped the proofreading process [9]. This is accomplished through the interaction of protein heterodimers with the DNA sequence containing the mismatch. Other dimers (e.g., MutL or MutH) are then recruited and interact with the DNA to initiate the repair signaling. When DNA structural integrity is compromised, specific genes and proteins are activated. One of such groups is 
the MutS family of proteins, integrated by MSH2, MSH3, and MSH6. These proteins are highly conserved between species and were originally found in Escherichia coli [10]; they have received great attention in recent years due to their relationship with several degenerative and pathologic diseases in humans. MSH proteins recognize errors in the genome sequence during replication, preventing the duplication of the damaged strand and repairing single strand breaks $[11,12]$. MSH2 either binds to MSH6 in the presence of base mispairing and other mismatches forming the MutS $\alpha$ heterodimer (MSH2-MSH6), or it binds to MSH3 in the presence of base deletions, generating the MutS $\beta$ heterodimer (MSH2-MSH3) [13, 14] (Figures 1(a) and 1(b)). The MutS $\alpha$ heterodimer then binds to the altered region and recruits the MutL family proteins, such as MLH1 and PMS2 (as a MutL $\alpha$ heterodimer). MLH1 and PMS2 in turn engage the enzymes needed for the DNA mismatch repair (Figure 1(c)). The DNA-MMR complex initiates the signaling process to replace the DNA altered region through the action of DNA polymerase $\delta$ and DNA ligase I $[15,16]$ (Figure 1(d)). The mechanism that recruits MMR proteins is ATP dependent [17].

Additionally, the activity of the two MutS dimers at the DNA mismatch site is dependent on interactions with the proliferating cell nuclear antigen (PCNA) [18, 19], which is an important cofactor that participates in both DNA replication and repair mechanisms. PCNA interacts with the MutS $\alpha$ dimer through its MSH6 domain and the MutS $\beta$ dimer binds with it at a region near the domain of MSH3 [20] (Figure 1(C)). When the basic human MMR system was reconstituted, the components purified were recombinant Mut $\alpha \alpha$ or MutS $\beta$, MutL $\alpha$, Exonuclease I (EXOI), PCNA, replication factor C (RFC, which loads PCNA onto DNA), the single-strand binding factor replication protein A (RPA), polymerase $\delta$, and DNA ligase I [21, 22]. In this system, the $5^{\prime}$-to- $3^{\prime}$ mismatch-directed strand excision required only MutS $\alpha$, EXOI, and RPA, whereas processing of substrates carrying a $3^{\prime}$ nick required also MutL $\alpha, \mathrm{PCNA}$, and RFC [23].

\section{Experimental Models Related with DNA MMR Deficiency}

MSH2 deficiency has been associated with frame-shift mutations known as microsatellite regions. These are specific DNA regions of tandem repeated units from one to six nucleotides. Microsatellites are found in centromeres, telomeres, promoters, and transcribed genes segments, causing DNA instability by inserting their repeated units and expanding the genomic sequence. This change in the original DNA sequence due to microsatellites can induce mutations within the genetic sequence, when mismatch bases are not corrected and copied to during DNA replication. Hence, microsatellite instability (MSI) has been widely used as a biochemical marker in clinical and laboratory tests for aging-related diseases and malignancies [24, 25].

MMR pathways have been studied in recent years because of their medical importance in several degenerative and pathological diseases in humans [26]. A positive correlation between MMR proteins deficiency, MSI and the malfunction of different processes, and regulatory mechanisms in cells has been established $[27,28]$.

\section{DNA MMR System and Cell Cycle Arrest Process}

Post-replicative DNA-MMR systems are critical during the response against diverse damaging stimuli that affect DNA fidelity, such as oxidative stress, xenobiotics, radiation, and so forth [28]. It has been corroborated that $\mathrm{MSH} 2$ participates in cell cycle arrest and apoptosis by different pathways, depending on the extent of DNA damage. Experiments conducted in $m s h 2-/-$ nontumor human lymphoblastoid cell lines have shown that the MMR system promotes G2/M arrest after UV-B induced DNA damage, and that a deficiency in MSH2 leads to a decrease in the induction of G2/M cell cycle checkpoint following this type of radiation. MSH2 proficient human lymphoblastoid cell lines cells also increase cell cycle checkpoint proteins levels such as CHK1, CDC25C, and CDC2, when compared with MSH2-deficient cells, after UV-B radiation treatment [29]. These proteins, when phosphorylated at specific amino acidic residues (Ser345, Ser216, Tyr15, and Thr14), regulate cell cycle control and inhibit cells from dividing until the DNA alterations are corrected, in accordance with MMR system's capacity to induce arrest in proliferative cells $[30,31]$. In another study, it was shown that MSH2 deficient nonmalignant mouse fibroblasts were partially resistant to UV-B-induced apoptosis and showed reduced S-Phase accumulation; when msh2 was modified by a point mutation that affected its expression, it was unable to participate in UV-B-induced apoptosis [32].

It has also been shown that UV-B induced DNA damage increases MSH6 protein levels in a p53 nondependent pathway. But in $m s h 6-1-$ primary mouse embryonic fibroblasts cells repair response to this type of damage was found to be significantly less sensitive to UV-B radiation cytotoxic effects as explained by a reduction in MSH6 protein levels. Therefore, MSH6 deficient cells were significantly less sensitive to the UV-B radiation cytotoxic effects and underwent significantly less apoptosis following irradiation than MSH6 proficient cells, thus indicating that UV-Binduced apoptosis was partially dependent on MSH6 levels [33]. These experiments suggest that MSH2 modulates both cell cycle regulation and apoptosis through independent and uncoupled mechanisms.

MMR proteins are also able to repair DNA through homologous recombination, a mechanism that repairs double-strand breaks using perfectly matched nucleotide sequences between two DNA strands. Both genomic and mitochondrial DNA sequences are exchanged through breaking and rejoining by specific protein complexes. The efficiency of homologous recombination depends on the length of uninterrupted sequence identity, as well as on the percentage of sequence identity within the region of homology [34]. These experiments suggest that MSH2 modulates both cell cycle regulation and apoptosis, through independent and uncoupled mechanisms. 


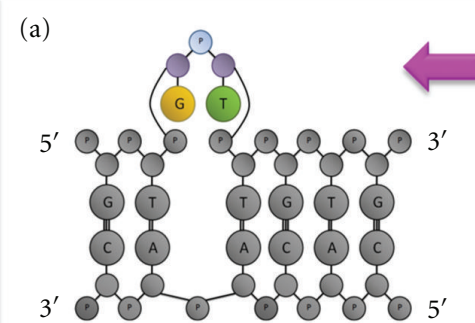

- Possible nucleotide insertion by strand slippage.

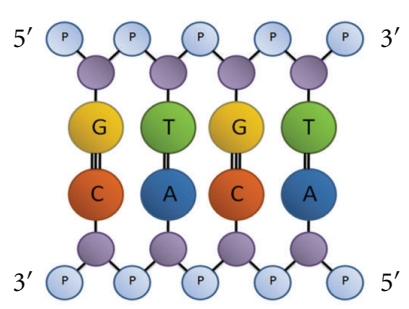

Normal DNA double-helix

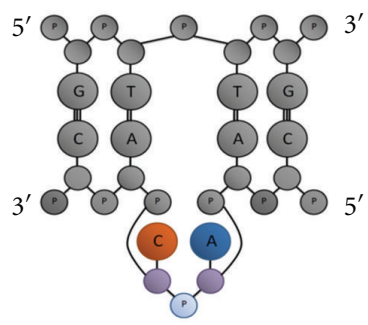

- Possible nucleotide deletion by strand slippage.

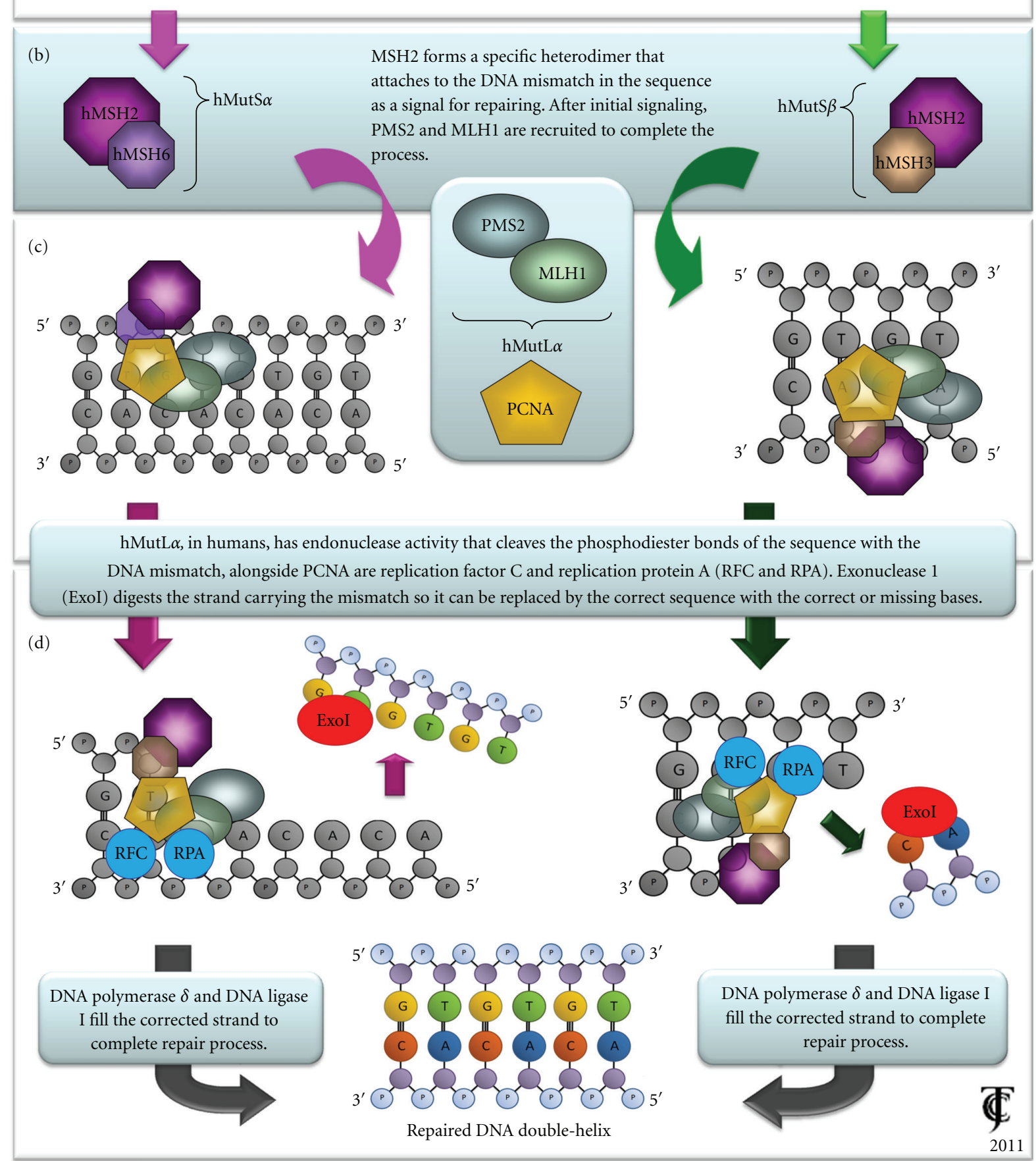

FIGURE 1: General DNA repair mechanisms mediated through MMR proteins MSH2, MSH6, and MSH3. Depending on the specific type of lesion in the genomic sequence, MSH protein family can initiate repair signaling pathways for maintaining genome integrity and fidelity. 


\section{MMR Deficiency Associated with Aging and Senescence}

When deficient DNA repair pathways, such as MMR system, do not detect altered DNA sequences, cell signaling pathways, as well as cell homeostasis, become unstable because DNA fidelity is compromised. Recent data have established a relationship between damaging stimuli, DNA lesions, and aging, with the absence or decrease in DNA repair systems [35]. It has been determined that $\mathrm{MSH} 2$ and $\mathrm{MLH} 1$ respond to oxidative DNA damage induced by UV-A radiation [36], and that MSH2 malfunction promotes degenerative conditions that increase with age and affect cell cycle and viability.

MMR efficiency has been studied in the detection of DNA-induced damage through cytotoxic compounds, such as cisplatin, used for chemotherapy in cancer patients, and it has been suggested that MSH2, besides its repair function, is able to induce programmed cell death, reducing abnormal cells in organisms. MSH2's role in signaling DNA damage-induced apoptosis has been recently demonstrated by Negureanu and Salsbury, along with confirmation that MMR defects alone can trigger tumorigenesis [37]. Stage II-III colorectal cancer patients, whose tumors retain DNA MMR activity, have shown benefits and better prognosis when 5-fluorouracil, a cytotoxic compound that induces DNA damage, is used; but patients with tumors that lost MMR activity do not [38]. In another study, MSH2 activity was tested on Trypanosoma cruzi and Trypanosoma brucei msh2-mutant strains, and it was shown that MMR system can also repair DNA lesions caused by oxidative stress [39]. Mutant NER-deficient XP-A fibroblasts were shown to have an increase in $\mathrm{CG}>\mathrm{TT}$ transitions in methylated $\mathrm{CpG}$ islands as a result of prolonged oxidative stress, compared with control fibroblasts with an active NER system [40]. Estes and collaborators [41] using a Caenorhabditis elegans msh2 knock-out model demonstrated that both fertility and survival rate were severely compromised; the life expectancy of these mutants also decreased significantly due to irreversible physiological damage. In another animal model, using CD-1 female mice, physiological stress related to breeding, methylation levels increased in msh 2 promoter region associated to age, ending ultimately in the accumulation of DNA damage markers due to MSH2 reduced expression [42]. All the previous evidence supports the idea that proficiency of MMR-system protects the cells from the early tumor occurrence.

Besides apoptosis, premature senescence has been proposed as another way in which cellular homeostasis is maintained. Damaged cells that undergo senescence do not proliferate and therefore the cellular damage can be stopped. However, studies that correlate MMR systems with the phenomenon of senescence are few. Some experiments conducted in young and senescent colonic and human embryonic lung fibroblast cell lines (CCD-18Co and IMR90, respectively) showed that MMR activity was significantly reduced in the senescent phenotype. In particular, MSH2 and MSH6 proteins were markedly downregulated in senescent cells. However, purified MSH2 and MSH6 extracts that were added to senescent cells led to the restoration of MMR activity. Semiquantitative RT-PCR analyses also showed that MSH2 mRNA levels were reduced in senescent cells from the same fibroblast cell lines mentioned before [43]. This evidence suggests that MMR system activity decreases during the senescent state, which could be interesting while studding other processes related to the aging process [44].

\section{Telomere Shortening and Senescence Related with MMR Deficiency}

Classic replicative senescence involves cell cycle arrest due to telomere shortening. Telomeres are protective structures at the ends of chromosomes that consist of tandem TTAGGG repeats bound to a protein complex known as shelterin [45]. Mouse embryonic fibroblasts (MEFs) deficient in $m s h 2$ expression showed a significant increase in chromosome aneuploidy, centrosome amplification, and defective mitotic spindle organization and unequal chromosome segregation [46]. Studies conducted in several models demonstrate that MMR system can regulate telomere maintenance and, therefore, alter cell senescence. In msh 2 knock-out mouse tissues and primary MEFs there was no apparent change in telomerase activity, telomere length, or recombination at telomeres. However, msh2 knockout MEFs presented chromosome ending without detectable telomeric DNA which correlated with an increase in chromosome end-toend fusions [47]. Interestingly, it was reported that mutations in MMR proteins in yeast, particulary MSH2, rescued telomerase-deficient strains survival, apparently by allowing telomere stabilization through an independent telomerase mechanism known as "alternating lengthening of telomeres" (ALT), which uses recombination-based pathways to maintain telomere length [48]. However, when this was studied in a mammalian in vivo model, by generating double mutant mice for telomerase and PMS2 (a MutL homolog), the previously proposed role for MMR genes in controlling telomere recombination and telomere length was not observed [49]. Similar results were reported in experiments with human colorectal, endometrial, and ovarian tumors, with or without MMR activity, indicating that in mammals the ALT mechanism is not essential during telomere maintenance $[47,49]$; nevertheless MMR may be important in detecting DNA damage induced by radiation, cytotoxic compounds, and oxidative stress, and depending on the damage extent, might be linked to cellular fate determination, leading either to apoptosis or to senescence, and therefore is important during tumorogenesis [50].

\section{Pathologies Related to MMR System Failure in Humans}

As discussed above, the MMR system is responsible for preserving DNA integrity and stability upon exposure to different damaging stimuli. If the MMR system fails, cell structure and functions will probably be affected, resulting in immortalization, malignant transformation, tumorigenesis, and/or degenerative diseases. One of such diseases is the Muir-Torre syndrome, a rare autosomal dominant disorder, 
which is characterized by the predisposition to both sebaceous neoplasms and internal malignancies. This condition is caused by an inherited germ-line mutation in one allele of MMR genes $m s h 2$ and $m l h 1$, leading to MMR deficiency. This condition causes MSI which in turn leads to DNA mutations [51-53].

Approximately 15\% of colorectal cancers display MSI in one or more MMR genes: mlh1, msh2, msh6, or pms2 [54]. Lynch syndrome, also called non-polyposis colorectal cancer and the most common form of inherited colorectal cancer, is a good example of a malignant disease associated to MSI. It develops in $60 \%$ of the patients with MMR gene mutations, commonly before 50 years of age $[51,55]$. Muir-Torre, as well as Lynch syndrome, is a condition caused by decreased DNA-MMR activity. Both diseases are characterized by high levels of MSI, supporting the fact that microsatellites are considered excellent markers to detect failures in DNA integrity maintenance [56].

The Ontario Familial Colorectal Cancer Registry is a population-based study of Lynch syndrome occurrence in relation to age, sex, and mutated genes [57]. This study indicated that the cumulative risk for colorectal cancer rose in patients of 70 years old or more, compared with the risk found in patients between 30 to 50 years old. This increase of cumulative risk was correlated with an increase in MSH2 and MLH1 mutations in 70 years patients. The male patients in this particular study presented more mutations in the mlh 1 gene compared with the female patients in all 3 age-dependent groups. However, female patients showed increased mutation levels in the msh 2 gene compared with the male patients, once again in all 3 age-dependent groups. These observations suggest that when genes responsible for maintaining DNA integrity, such as $m s h 2$ and $m l h 1$, are mutated, and their capacity to repair DNA decreases, the accumulation of mutations and strand breaks may lead to aging and disease. In other words, when the organisms fail to maintain their homeostasis, they become susceptible to aging and degenerative diseases. Although some of these mutations may be inherited, exposure to toxic and damaging insults like ROS, chemicals, and radiation can abruptly alter or accelerate their formation.

Cell-microorganism interactions can further generate failure of MMR system activity. One example is the mucosaassociated bacteria that significantly contribute to the colonic environment in humans. Enteropathogenic Escherichia coli (EPEC) carry the eae gene, which encodes the bacterial adhesion protein intimin; this protein enables bacteria to attach to the intestinal epithelium by using a type III secretion system which translocates effector proteins into host cells. These bacteria can influence molecular pathways involved in colorectal tumorigenesis. The ability of EPEC to downregulate DNA-MMR proteins such as MSH2 and MLH1 demonstrates that environmental factors can increase tumorigenesis in colonic epithelial cells [58]. Other tumorigenic processes resulting from MMR system failure are melanoma and breast cancer. MSI and mutations in MMR genes contribute significantly to both conditions in human patients, confirming MMR potential role in carcinoma susceptibility $[59,60]$. Another gene associated with MMR defects that has been extensively studied is the tumorsuppressor gene BRCA1 [61] related to breast cancer. BRCA1 encodes a protein that repairs damaged DNA and initiates the apoptotic process. Errors in BRCA1 expression result in unrepaired DNA [62]. Such mutations cause uncontrollable cell growth resulting in tumor formation. In experimental trials, the role of the BRCA1 protein in modulating a 6thioguanine (6-TG) induced MMR damage response was studied, using an isogenic human breast cancer cell line model, including a BRCA1 mutated cell line (HCC1937). The results showed that the tumor cells were more resistant to chemotherapeutic drugs, like 6-thioguanine, as manifested by a reduced G2/M arrest and decreased apoptosis [63]. In this context, BRCA1-mutant cells are more resistant to damage by this drug, than BRCA1-positive cells. This might suggest a probable mechanism by which the MMR system may regulate cell cycle and apoptosis, in correlation with the functionality of tumor suppressing genes like BRCA1 [64].

\section{The Use of MMR to Study Aging: The Bats Example}

As mentioned before, the accumulation of DNA damage is known to induce alterations in the cell's mechanisms and structure that might lead to aging; and since DNAMMR system is highly conserved between species, our group decided to determine the importance of this system during the aging process in a different animal model. Bats (class: Mammalia, order: Chiroptera) are an interesting model due to their high longevity and particular resistance to adverse environmental factors [65], and therefore, the levels of MMR proteins MSH2 and MLH1, as well as microsatellite markers were quantified, in liver, lung, and brain of young, adult, and old bats, both female and male. Individuals of two different wild bats species were used: Myotis velifer and Desmodus rotundus. Catalase, glutathione peroxidase, and superoxide dismutase were also analyzed to determine if the antioxidant protection negatively correlated with DNA damage [66]. The first species, the cave Myotis, is an insectivorous bat that has an average lifespan of 8 to 12 years ( 11.3 years in the field) based on mark-recapture studies and an average adult weight of $10.1 \mathrm{~g}$ [67] which migrates to higher altitudes to mate and hibernate $[68,69]$. On the other hand, the vampire bat $D$. rotundus has an average lifespan of 12 to 20 years [70] and an average adult weight of $32.2 \mathrm{~g}$ [67]. Vampire bats feed on the blood of stock animals and do not undergo hibernation $[71,72]$. The study conducted determined that $M$. velifer (the short lived species) presented decreased levels of MSH2 and MLH1 with increased MSI (Figure 2(a)) and reduced antioxidant activity as these insectivorous bats became older. However, in the case of $D$. rotundus (the long lived species), there was a reduced MSI in the vampire bats from adult to old age, compared with the young control bats, which correlated with reduced levels of MSH2 and MLH1 (Figure 2(b)) along with improved antioxidant activities in the old bats that matched the activities seen in the adult groups from the other species [66]. An important feature of this study was the demonstration that the analysis of MMR deficiency and 

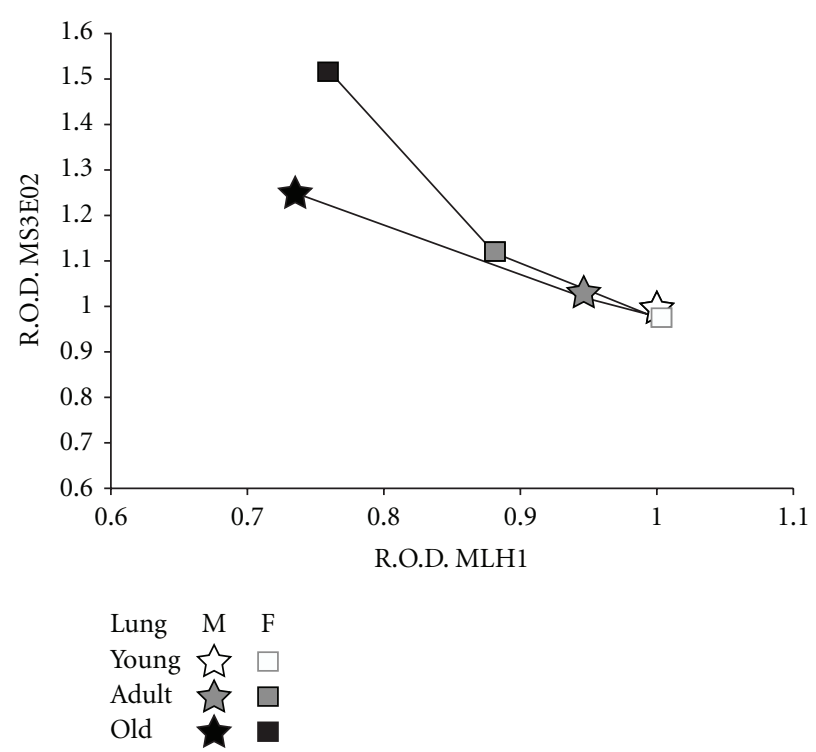
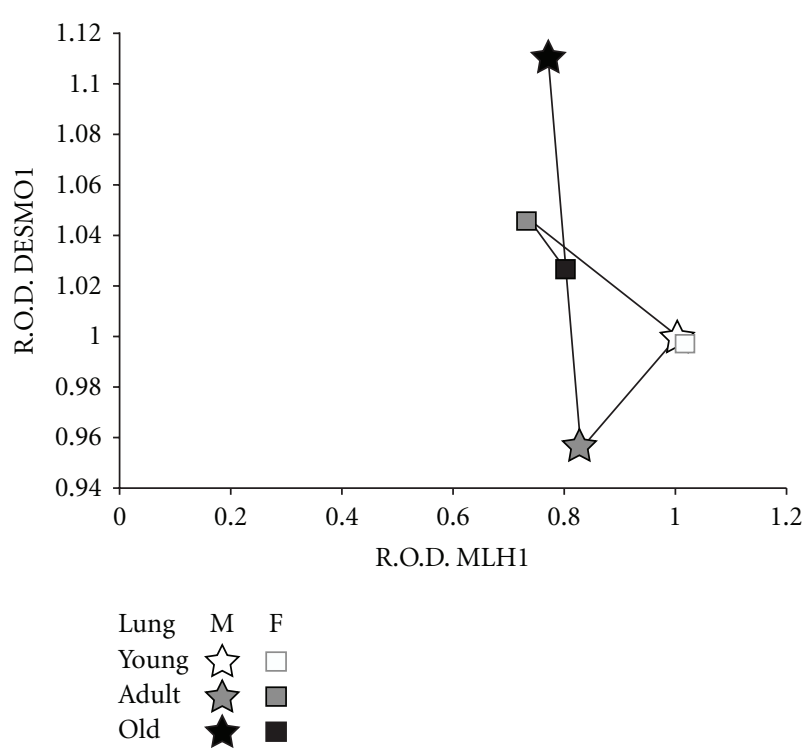

(a)

(b)

FIgURE 2: Comparisons between MLH1 levels and microsatellite instability. The figure shows the correlations between MSI and MLH1 protein levels, found in the female (F) and male (M) bat lung samples. The correlations were performed considering the relative optical densities (RODs) obtained from the densitometries of the PCR products from DNA microsatellite sequences (MS3EO2 for M. velifer and DESMO1 for D. rotundus), and the MLH1 protein levels were determined by Western Blot analysis in both species. Young bats were given the arbitrary value of one, in order to compare them with the other age groups. Each age group consisted of 5 invidious $(n=5)$ either male or female bats. One-way ANOVA tests, followed by Tukey-Kramer variance analyses, were used to compare among groups, by sex and age. A 0.05 level of probability was used as a minimum criterion of significance in all analyses. The figures correspond to: (a) Myotis velifer (MS3EO2 versus MLH1) and (b) Desmodus rotundus (DESMO1 versus MLH1).

the variations of MSI are an excellent model that can be used in aging studies to correlate Harman's theory of aging with processes related to DNA damage.

\section{Final Considerations}

Although most of the studies related to the MMR deficiency and genomic alterations are associated with carcinogenic processes, there is a high prospective in using these tools for aging and senescence studies. Studying MMR impairment could also be used to correlate cancer and aging. It is known that cancer predisposition is more frequent when DNA repair is deteriorated in the elderly, which correlates with DNA damage accumulation (e.g., MSI) throughout lifetime, and it might explain the origin of these diseases as proposed by Harman [1, 73]. However, mutations in MMR proteins have been related with other malignancies found not only in elderly patients, but also in children, such as haematological malignancies, brain tumors, Lynch syndrome, and neurofibromatosis [74, 75]. Therefore it would be important to determine which of these malignant conditions are exclusively age-dependent processes or solely DNA damage/mutation-dependent. Another issue to consider is the interaction with other proteins complexes that could assist in the MMR system. It was recently determined in msh2-knockout mouse embryonic fibroblasts, HEK, and HeLa cell lines that MSH2 is also in charge of the recruitment of the ATR protein, which is in charge, along with ATM protein, of the DNA damage response signaling, frequently associated with cell death by apoptosis. According to this study ATR is recruited by two different pathways: one of which is mediated by RPA (replication protein A) and relays a signal to the Chk1 protein, whereas the other pathway depends on $\mathrm{MSH} 2$ and is responsible for Chk2 activation [76]. Further investigations could be benefited from experiments based on correlations between MMR deficiencies, DNA damage markers in new models such as bats, since they have prolonged longevities that might increase our understanding of $\mathrm{MSH} 2$ pathways, revealing new processes that regulate cellular homeostasis by maintaining DNA integrity.

\section{Acknowledgments}

The authors would like to acknowledge Dr. Banalata Sen from the National Institute of Environmental Health Sciences and Dr. Carmen Ríos from the Barshop Institute for Aging and Longevity Studies-UTHSCSA for their critical reading and edition of the paper. Mr. Joel Kipi Turok for English editing; Dr. H. González-Márquez, UAM-I, for his help with Figure 1. This work was supported by CONACyT Grant no. CB-2006-1-59659. J. C. Conde-Pérezprina is a CONACyT scholarship holder.

\section{References}

[1] D. Harman, "The free radical theory of aging," Antioxidants and Redox Signaling, vol. 5, no. 5, pp. 557-561, 2003. 
[2] B. Halliwell and J. M. C. Gutteridge, Free Radicals in Biology and Medicine, Oxford University Press, New York, NY, USA, 3rd edition, 2004.

[3] A. M. Florea, E. N. Yamoah, and E. Dopp, "Intracellular calcium disturbances induced by arsenic and its methylated derivatives in relation to genomic damage and apoptosis induction," Environmental Health Perspectives, vol. 113, no. 6, pp. 659-664, 2005.

[4] S. E. Boley, V. A. Wong, J. E. French, and L. Recio, "p53 heterozygosity alters the mRNA expression of p53 target genes in the bone marrow in response to inhaled benzene," Toxicological Sciences, vol. 66, no. 2, pp. 209-215, 2002.

[5] R. Barouki, "Ageing free radicals and cellular stress," Medecine/Sciences, vol. 22, no. 3, pp. 266-272, 2006.

[6] E. Moustacchi, "DNA damage and repair: consequences on dose-responses," Mutation Research, vol. 464, no. 1, pp. 35-40, 2000.

[7] C. Bernstein, H. Bernstein, C. M. Payne, and H. Garewal, "DNA repair/pro-apoptotic dual-role proteins in five major DNA repair pathways: fail-safe protection against carcinogenesis," Mutation Research, vol. 511, no. 2, pp. 145-178, 2002.

[8] T. Nakano, A. Katafuchi, H. Terato, T. Suzuki, B. Van Houten, and H. Ide, "Activity of nucleotide excision repair enzymes for oxanine cross-link lesions," Nucleic Acids Symposium Series, no. 49, pp. 293-294, 2005.

[9] T. A. Kunkel and D. A. Erie, "DNA mismatch repair," Annual Review of Biochemistry, vol. 74, pp. 681-710, 2005.

[10] J. Jiricny, "MutL $\alpha$ : at the cutting edge of mismatch repair," Cell, vol. 126, no. 2, pp. 239-241, 2006.

[11] D. Kültz, "Molecular and evolutionary basis of the cellular stress response," Annual Review of Physiology, vol. 67, pp. 225257, 2005.

[12] D. N. Mullins, E. L. Crawford, S. A. Khuder, D. A. Hernandez, Y. Yoon, and J. C. Willey, "CEBPG transcription factor correlates with antioxidant and DNA repair genes in normal bronchial epithelial cells but not in individuals with bronchogenic carcinoma_aptad 20050829," BMC Cancer, vol. 5, article 141, 2005.

[13] P. Peltomäki, "Deficient DNA mismatch repair: a common etiologic factor for colon cancer," Human Molecular Genetics, vol. 10, no. 7, pp. 735-740, 2001.

[14] S. Santucci-Darmanin and V. Paquis-Flucklinger, "Homologs of MutS and MutL during mammalian meiosis," Medecine Sciences, vol. 19, no. 1, pp. 85-91, 2003.

[15] Q. H. Phung, D. B. Winter, R. Alrefai, and P. J. Gearhart, "Cutting edge: hypermutation in Ig V genes from mice deficient in the MLH1 mismatch repair protein," Journal of Immunology, vol. 162, no. 6, pp. 3121-3124, 1999.

[16] H. Saribasak, D. Rajagopal, R. W. Maul, and P. J. Gearhart, "Hijacked DNA repair proteins and unchained DNA polymerases," Philosophical Transactions of the Royal Society B, vol. 364, no. 1517, pp. 605-611, 2009.

[17] H. Geng, M. Sakato, V. DeRocco et al., "Biochemical analysis of the human mismatch repair proteins hMutS $\alpha \mathrm{MSH} 2^{G 674 A}$ MSH6 and MSH2-MSH6 ${ }^{\text {T1219D }}$," Journal of Biological Chemistry, vol. 287, no. 13, pp. 9777-9791, 2012.

[18] Z. Hong, J. Jiang, K. Hashiguchi, M. Hoshi, L. Lan, and A. Yasui, "Recruitment of mismatch repair proteins to the site of DNA damage in human cells," Journal of Cell Science, vol. 121, no. 19, pp. 3146-3154, 2008.

[19] S. V. Mudrak, C. Welz-Voegele, and S. Jinks-Robertson, "The polymerase $\eta$ translesion synthesis DNA polymerase acts independently of the mismatch repair system to limit mutagenesis caused by 7,8-dihydro-8-oxoguanine in yeast,"
Molecular and Cellular Biology, vol. 29, no. 19, pp. 5316-5326, 2009.

[20] F. J. López de Saro, "Regulation of interactions with sliding clamps during DNA replication and repair," Current Genomics, vol. 10, no. 3, pp. 206-215, 2009.

[21] Y. Zhang, F. Yuan, S. R. Presnell et al., "Reconstitution of 5' directed human mismatch repair in a purified system," Cell, vol. 122, no. 5, pp. 693-705, 2005.

[22] N. Constantin, L. Dzantiev, F. A. Kadyrov, and P. Modrich, "Human mismatch repair: reconstitution of a nick-directed bidirectional reaction," Journal of Biological Chemistry, vol. 280, no. 48, pp. 39752-39761, 2005.

[23] L. Dzantiev, N. Constantin, J. Genschel, R. R. Iyer, P. M. Burgers, and P. Modrich, "A defined human system that supports bidirectional mismatch-provoked excision," Molecular Cell, vol. 15, no. 1, pp. 31-41, 2004.

[24] Y. Lin and J. H. Wilson, "Diverse effects of individual mismatch repair components on transcription-induced CAG repeat instability in human cells," DNA Repair, vol. 8, no. 8, pp. 878-885, 2009.

[25] H. Alazzouzi, E. Domingo, S. González et al., "Low levels of microsatellite instability characterize MLH1 and MSH2 HNPCC carriers before tumor diagnosis," Human Molecular Genetics, vol. 14, no. 2, pp. 235-239, 2005.

[26] P. Modrich and R. Lahue, "Mismatch repair in replication fidelity, genetic recombination, and cancer biology," Annual Review of Biochemistry, vol. 65, pp. 101-133, 1996.

[27] A. D. Auerbach and P. C. Verlander, "Disorders of DNA replication and repair," Current Opinion in Pediatrics, vol. 9, no. 6, pp. 600-616, 1997.

[28] Z. Yang, R. Lau, J. L. Marcadier, D. Chitayat, and C. E. Pearson, "Replication inhibitors modulate instability of an expanded trinucleotide repeat at the myotonic dystrophy type 1 disease locus in human cells," American Journal of Human Genetics, vol. 73, no. 5, pp. 1092-1105, 2003.

[29] K. A. Narine, K. E. Felton, A. A. Parker, V. A. Tron, and S. E. Andrew, "Non-tumor cells from an MSH2-null individual show altered cell cycle effects post-UVB," Oncology Reports, vol. 18, no. 6, pp. 1403-1411, 2007.

[30] C. S. Sørensen, L. T. Hansen, J. Dziegielewski et al., “The cell-cycle checkpoint kinase Chk1 is required for mammalian homologous recombination repair," Nature Cell Biology, vol. 7, no. 2, pp. 195-201, 2005.

[31] V. Leung-Pineda, C. E. Ryan, and H. Piwnica-Worms, "Phosphorylation of Chk1 by ATR is antagonized by a chk1regulated protein phosphatase 2A circuit," Molecular and Cellular Biology, vol. 26, no. 20, pp. 7529-7538, 2006.

[32] M. Seifert, S. J. Scherer, W. Edelmann et al., "The DNAmismatch repair enzyme hMSH2 modulates UV-B-induced cell cycle arrest and apoptosis in melanoma cells," Journal of Investigative Dermatology, vol. 128, no. 1, pp. 203-213, 2008.

[33] L. C. Young, K. J. Thulien, M. R. Campbell, V. A. Tron, and S. E. Andrew, "DNA mismatch repair proteins promote apoptosis and suppress tumorigenesis in response to UVB irradiation: an in vivo study," Carcinogenesis, vol. 25, no. 10, pp. 1821-1827, 2004.

[34] M. Kappeler, E. Kranz, K. Woolcock, O. Georgiev, and W. Schaffner, "Drosophila bloom helicase maintains genome integrity by inhibiting recombination between divergent DNA sequences," Nucleic Acids Research, vol. 36, no. 21, pp. 69076917, 2008.

[35] A. M. Skinner and M. S. Turker, "Oxidative mutagenesis, mismatch repair, and aging," Science of Aging Knowledge Environment, vol. 2005, no. 9, article re3, 2005. 
[36] P. Pitsikas, D. Lee, and A. J. Rainbow, "Reduced host cell reactivation of oxidative DNA damage in human cells deficient in the mismatch repair gene hMSH2," Mutagenesis, vol. 22, no. 3, pp. 235-243, 2007.

[37] L. Negureanu and F. R. Salsbury Jr., "The molecular origin of the MMR-dependent apoptosis pathway from dynamics analysis of MutS $\alpha$-DNA complexes," Journal of Biomolecular Structure \& Dynamics, vol. 30, no. 3, pp. 347-361, 2012.

[38] M. Iwaizumi, S. Tseng-Rogenski, and J. M. Carethers, "DNA mismatch repair proficiency executing 5-fluorouracil cytotoxicity in colorectal cancer cells," Cancer Biology and Therapy, vol. 12, no. 8, pp. 756-764, 2011.

[39] P. C. Campos, V. G. Silva, C. Furtado et al., “Trypanosoma cruzi MSH2: functional analyses on different parasite strains provide evidences for a role on the oxidative stress response," Molecular and Biochemical Parasitology, vol. 176, no. 1, pp. 816, 2011.

[40] D. H. Lee, T. R. O'Connor, and G. P. Pfeifer, "Oxidative DNA damage induced by copper and hydrogen peroxide promotes $\mathrm{CG} \rightarrow$ TT tandem mutations at methylated CpG dinucleotides in nucleotide excision repair-deficient cells," Nucleic Acids Research, vol. 30, no. 16, pp. 3566-3573, 2002.

[41] S. Estes, P. C. Phillips, D. R. Denver, W. K. Thomas, and M. Lynch, "Mutation accumulation in populations of varying size: the distribution of mutational effects for fitness correlates in caenorhabditis elegans," Genetics, vol. 166, no. 3, pp. 12691279, 2004.

[42] J. C. Conde-Pérezprina, A. Luna-López, N. E. LópezDiazguerrero, P. Damián-Matsumura, A. Zentella, and M. Königsberg, "Msh2 promoter region hypermethylation as a marker of aging-related deterioration in old retired female breeder mice," Biogerontology, vol. 9, no. 5, pp. 325-334, 2008.

[43] I. Y. Chang, M. Jin, P. Y. Sang et al., "Senescence-dependent MutS $\alpha$ dysfunction attenuates mismatch repair," Molecular Cancer Research, vol. 6, no. 6, pp. 978-989, 2008.

[44] L. C. Young, A. C. Peters, T. Maeda et al., "DNA mismatch repair protein msh6 is required for optimal levels of ultraviolet-b-induced apoptosis in primary mouse fibroblasts," Journal of Investigative Dermatology, vol. 121, no. 4, pp. 876-880, 2003.

[45] S. W. L. Chan and E. H. Blackburn, "New ways not to make ends meet: telomerase, DNA damage proteins and heterochromatin," Oncogene, vol. 21, no. 4, pp. 553-563, 2002.

[46] M. R. Campbell, Y. Wang, S. E. Andrew, and Y. Liu, "Msh2 deficiency leads to chromosomal abnormalities, centrosome amplification, and telomere capping defect," Oncogene, vol. 25, no. 17 , pp. 2531-2536, 2006.

[47] P. Martinez, I. Siegl-Cachedenier, J. M. Flores, and M. A. Blasco, "MSH2 deficiency abolishes the anticancer and proaging activity of short telomeres," Aging Cell, vol. 8, no. 1, pp. 2-17, 2009.

[48] A. Rizki and V. Lundblad, "Defects in mismatch repair promote telomerase-independent proliferation," Nature, vol. 411, no. 6838, pp. 713-716, 2001.

[49] I. Siegl-Cachedenier, P. Muñoz, J. M. Flores, P. Klatt, and M. A. Blasco, "Deficient mismatch repair improves organismal fitness and survival of mice with dysfunctional telomeres," Genes and Development, vol. 21, no. 17, pp. 2234-2247, 2007.

[50] I. Ibanez de Caceres, N. Frolova, R. J. Varkonyi et al., “Telomerase is frequently activated in tumors with microsatellite instability," Cancer Biology and Therapy, vol. 3, no. 3, pp. 289292, 2004.

[51] S. Perera, L. Ramyar, A. Mitri et al., "A novel complex mutation in MSH2 contributes to both Muir-Torre and Lynch
Syndrome," Journal of Human Genetics, vol. 55, no. 1, pp. 3741, 2010.

[52] A. Morales-Burgos, J. L. Sánchez, L. D. Figueroa et al., "MSH2 and MLH-1 Protein expression in muir torre syndromerelated and sporadic sebaceous neoplasms," Puerto Rico Health Sciences Journal, vol. 27, no. 4, pp. 322-327, 2008.

[53] D. Torre, "Multiple sebaceous tumors," Archives of Dermatology, vol. 98, no. 5, pp. 549-551, 1968.

[54] E. Vilar and S. B. Gruber, "Microsatellite instability in colorectal cancerthe stable evidence," Nature Reviews Clinical Oncology, vol. 7, no. 3, pp. 153-162, 2010.

[55] F. C. C. da Silva, M. D. Valentin, F. D. O. Ferreira, D. M. Carraro, and B. M. Rossi, "Mismatch repair genes in Lynch syndrome: a review," Sao Paulo Medical Journal, vol. 127, no. 1, pp. 46-51, 2009.

[56] P. M. Lynch, "The hMSH2 and hMLH1 genes in hereditary nonpolyposis colorectal cancer," Surgical Oncology Clinics of North America, vol. 18, no. 4, pp. 611-624, 2009.

[57] Y. H. Choi, M. Cotterchio, G. McKeown-Eyssen et al., "Penetrance of colorectal cancer among MLH1/MSH2 carriers participating in the colorectal cancer familial registry in Ontario," Hereditary Cancer in Clinical Practice, vol. 7, no. 1, article 14, 2009.

[58] O. D. K. Maddocks, A. J. Short, M. S. Donnenberg, S. Bader, and D. J. Harrison, "Attaching and effacing Escherichia coli downregulate DNA mismatch repair protein in vitro and are associated with colorectal adenocarcinomas in humans," PLoS ONE, vol. 4, no. 5, Article ID e5517, 2009.

[59] G. Ponti, L. Losi, G. Pellacani et al., "Malignant melanoma in patients with hereditary nonpolyposis colorectal cancer," British Journal of Dermatology, vol. 159, no. 1, pp. 162-168, 2008.

[60] S. A. Martin, A. McCarthy, L. J. Barber et al., "Methotrexate induces oxidative DNA damage and is selectively lethal to tumour cells with defects in the DNA mismatch repair gene MSH2," EMBO Molecular Medicine, vol. 1, no. 6-7, pp. 323337, 2009.

[61] F. Fostira, G. Thodi, I. Konstantopoulou, R. Sandaltzopoulos, and D. Yannoukakos, "Hereditary cancer syndromes," Journal of B.U.ON., vol. 12, no. 1, pp. S13-S22, 2007.

[62] A. Agarwal, S. Gupta, and R. K. Sharma, "Role of oxidative stress in female reproduction," Reproductive Biology and Endocrinology, vol. 3, article 28, 2005.

[63] K. Yamane, J. E. Schupp, and T. J. Kinsella, "BRCA1 activates a G2-M cell cycle checkpoint following 6-thioguanine-induced DNA mismatch damage," Cancer Research, vol. 67, no. 13, pp. 6286-6292, 2007.

[64] R. A. Jensen, M. E. Thompson, T. L. Jetton et al., "BRCA1 is secreted and exhibits properties of a granin," Nature Genetics, vol. 12, no. 3, pp. 303-308, 1996.

[65] G. Neuweiler, The Biology of Bats, Oxford University Press, New York, NY, USA, 2000.

[66] J. C. Conde-Pérezprina, A. Luna-López, V. Y. GonzálezPuertos, T. Zenteno-Savín, M. A. León-Galván, and M. Königsberg, "DNA MMR systems, microsatellite instability and antioxidant activity variations in two species of wild bats: myotis velifer and Desmodus rotundus, as possible factors associated withlongevity," Age. In press.

[67] G. S. Wilkinson and J. M. South, "Life history, ecology and longevity in bats," Aging Cell, vol. 1, no. 2, pp. 124-131, 2002.

[68] J. H. Fitch, K. A. Shump, and A. U. Shump, "Myotis velifer," Mammalian Species, vol. 149, pp. 1-5, 1981. 
[69] K. D. Jürgens and J. Prothero, "Scaling of maximal lifespan in bats," Comparative Biochemistry and Physiology A, vol. 88, no. 2, pp. 361-367, 1987.

[70] R. D. Lord, F. Muradali, and L. Lazaro, "Age composition of vampire bats (Desmodus rotundus) in Northern Argentina and Southern Brazil," Journal of Mammalogy, vol. 57, pp. 573$575,1976$.

[71] A. Balmori, "El estudio de los quirópteros a través de sus emisiones ultrasónicas," Galemys Boletín SECEM, vol. 10, pp. 12-19, 1998.

[72] A. K. Brunet-Rossinni and S. N. Austad, "Ageing studies on bats: a review," Biogerontology, vol. 5, no. 4, pp. 211-222, 2004.

[73] T. Arai, M. Sawabe, T. Hosoi, and N. Tanaka, "Role of DNA repair systems in malignant tumor development in the elderly," Geriatrics and Gerontology International, vol. 8, no. 2, pp. 65-72, 2008.

[74] K. Wimmer and J. Etzler, "Constitutional mismatch repairdeficiency syndrome: have we so far seen only the tip of an iceberg?" Human Genetics, vol. 124, no. 2, pp. 105-122, 2008.

[75] S. Krüger, M. Kinzel, C. Walldorf et al., "Homozygous PMS2 germline mutations in two families with early-onset haematological malignancy, brain tumours, HNPCC-associated tumours, and signs of neurofibromatosis type 1," European Journal of Human Genetics, vol. 16, no. 1, pp. 62-72, 2008.

[76] N. Pabla, Z. Ma, M. A. McIlhatton, R. Fishel, and Z. Dong, "hMSH2 recruits ATR to DNA damage sites for activation during DNA damage-induced apoptosis," Journal of Biological Chemistry, vol. 286, no. 12, pp. 10411-10418, 2011. 


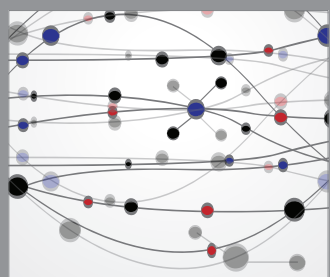

The Scientific World Journal
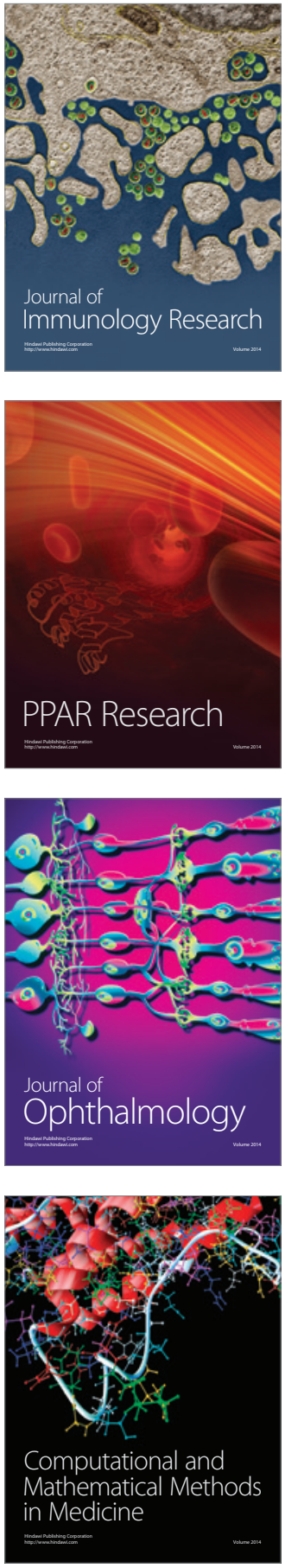

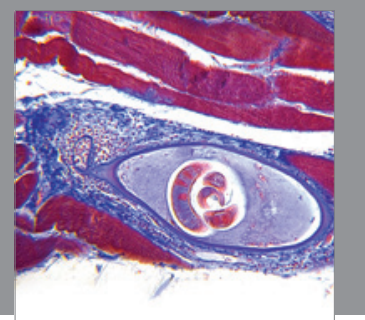

Gastroenterology

Research and Practice
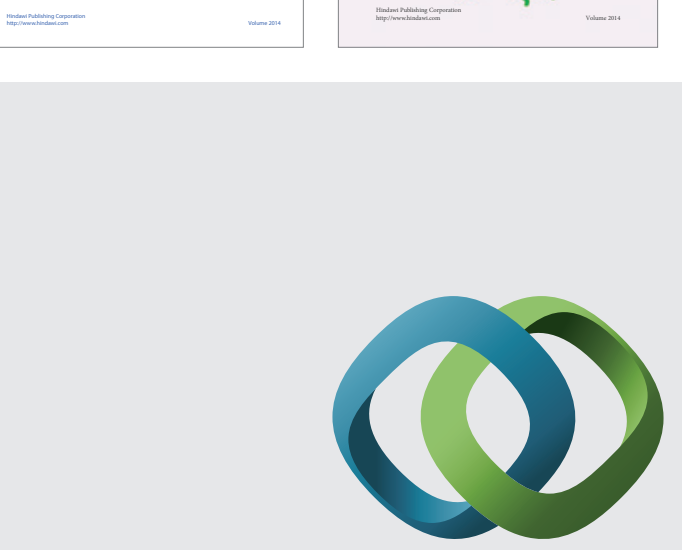

\section{Hindawi}

Submit your manuscripts at

http://www.hindawi.com
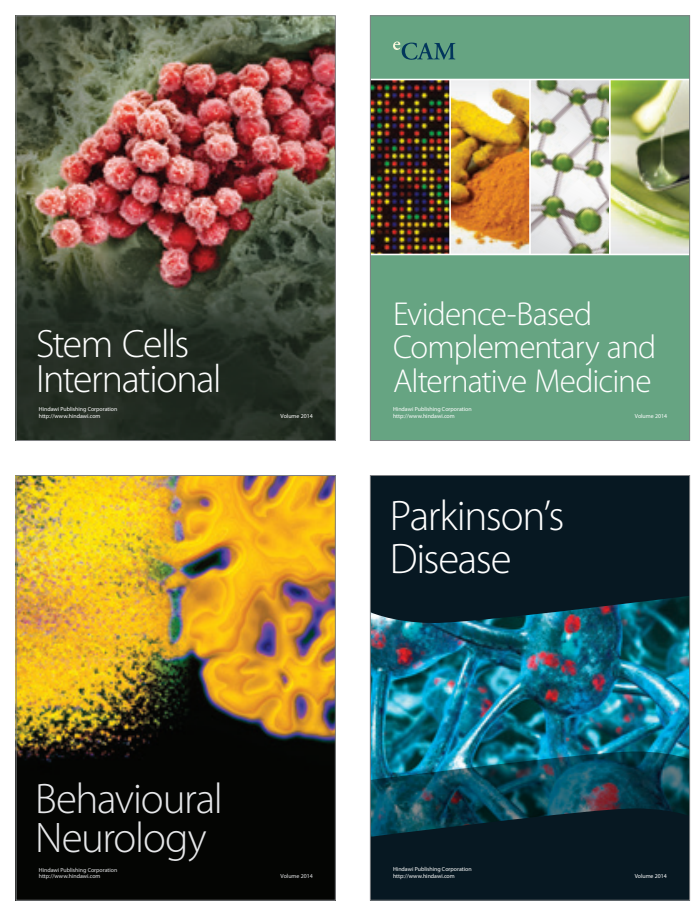

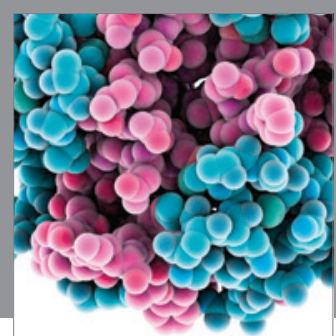

Journal of
Diabetes Research

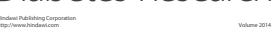

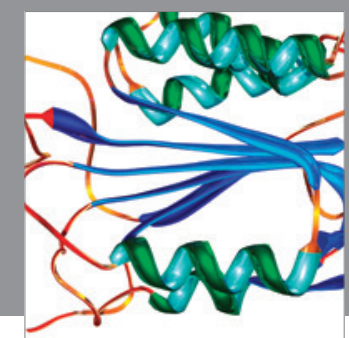

Disease Markers
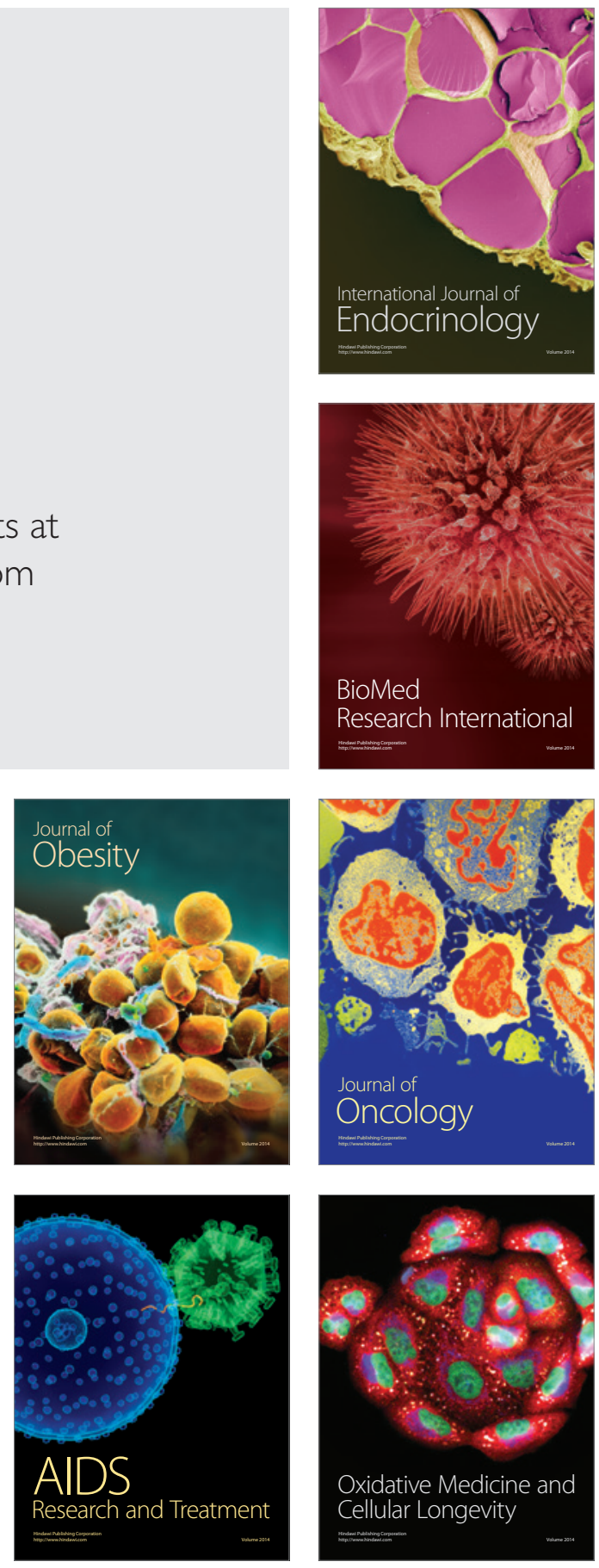